\title{
Life threatening cardiac arrhythmias following treatment of newly diagnosed Addison's disease
}

\author{
J Rochester ${ }^{1}$, D Aflorei ${ }^{1}$, A Roberts ${ }^{2}$ \& A Wilton ${ }^{1}$
}

Departments of Endocrinology ${ }^{1}$ \& Anaesthetics ${ }^{2}$, Ysbyty Gwynedd, Bangor, UK

GIG

The occurrence of long QT interval in Addison's disease was first described in $1950 .{ }^{1}$ The same author subsequently reported this same ECG abnormality in 32 of a group of 47 $(68 \%)$ patients with untreated Addison's disease. ${ }^{2}$

We have found 13 case reports of life threatening cardiac arrhythmias associated with long QT interval in secondary hypoadrenalism. In all cases the arrhythmias occurred prior to glucocorticoid treatment. We describe a case of life threatening arrhythmias associated with long QT interval occurring after treatment for primary adrenal insufficiency.

\section{Case History}

A 36 year old man presented with an episode of collapse following a 2 week history of weakness, fatigue and vomiting. He had $7 \mathrm{~kg}$ of weight loss over the preceding 6 months. Treatment with thyroxine $50 \mathrm{mcg} O D$ had been commenced in primary care 2 months earlier for hypothyroidism (Free T4 9.4pmol/L, TSH 8.4mU/L and anti-TPO antibodies 103.1 $\mathrm{IU} / \mathrm{ml})$.

Previous medical history was one of pulmonary sarcoidosis 6 years earlier which had resolved without treatment. Other than thyroxine he was not taking any regular medications and family history was unremarkable. Examination revealed cachexia (weight $53.6 \mathrm{Kg}$ ), vitiligo, sinus tachycardia $110 \mathrm{bpm}$ and hypotension $(90 / 66 \mathrm{mmHg})$. An initial diagnosis of sepsis with hypotensive collapse was revised to Addison's disease based on results of investigations.

\section{Initial Investigations}

\begin{tabular}{|l|l|}
\hline Sodium & $115 \mathrm{mmol} / \mathrm{L}$ \\
\hline Potassium & $4.4 \mathrm{mmol} / \mathrm{L}$ \\
\hline Urea & $9.9 \mathrm{mmol} / \mathrm{L}$ \\
\hline Creatinine & $81 \mathrm{mmol} / \mathrm{L}$ \\
\hline Magnesium & $0.91 \mathrm{mmol} / \mathrm{L}$ \\
\hline $\mathrm{pH}$ & 7.30 \\
\hline Bicarbonate & $11.3 \mathrm{mmol} / \mathrm{L}$ \\
\hline Cortisol & $166 \mathrm{nmol} / \mathrm{L}$ \\
\hline
\end{tabular}

Treatment in the acute situation was with intravenous normal saline and hydrocortisone $100 \mathrm{mg}$ STAT followed by $200 \mathrm{mg}$ over 24 hours. He then received maintenance treatment with oral hydrocortisone $10,5 \& 5 \mathrm{mg}$ plus fludrocortisone $100 \mathrm{mcg}$ once daily. This treatment resulted in rapid clinical improvement and normal electrolyte levels. Further investigation confirmed the diagnosis of primary hypoadrenalism.

\section{Endocrine Investigations}

9am cortisol $137 \mathrm{nmol} / \mathrm{L}$ and ACTH $1337 \mathrm{ng} / \mathrm{L}$

Aldosterone $<100 \mathrm{pmol} / \mathrm{L}$

Short Synacthen test cortisols:- 0 minute 137, 30 minutes 136, 60 minutes 142 nmol/L

Adrenal auto-antibodies positive

Shortly before discharge at 6 days he sustained a cardiac arrest with polymorphic ventricular tachycardia (torsades de pointes), ventricular tachycardia and ventricular fibrillation requiring DC cardioversion 27 times.

\section{ECGs}

1. ECG 6 years earlier - QTC $402 \mathrm{msec}$

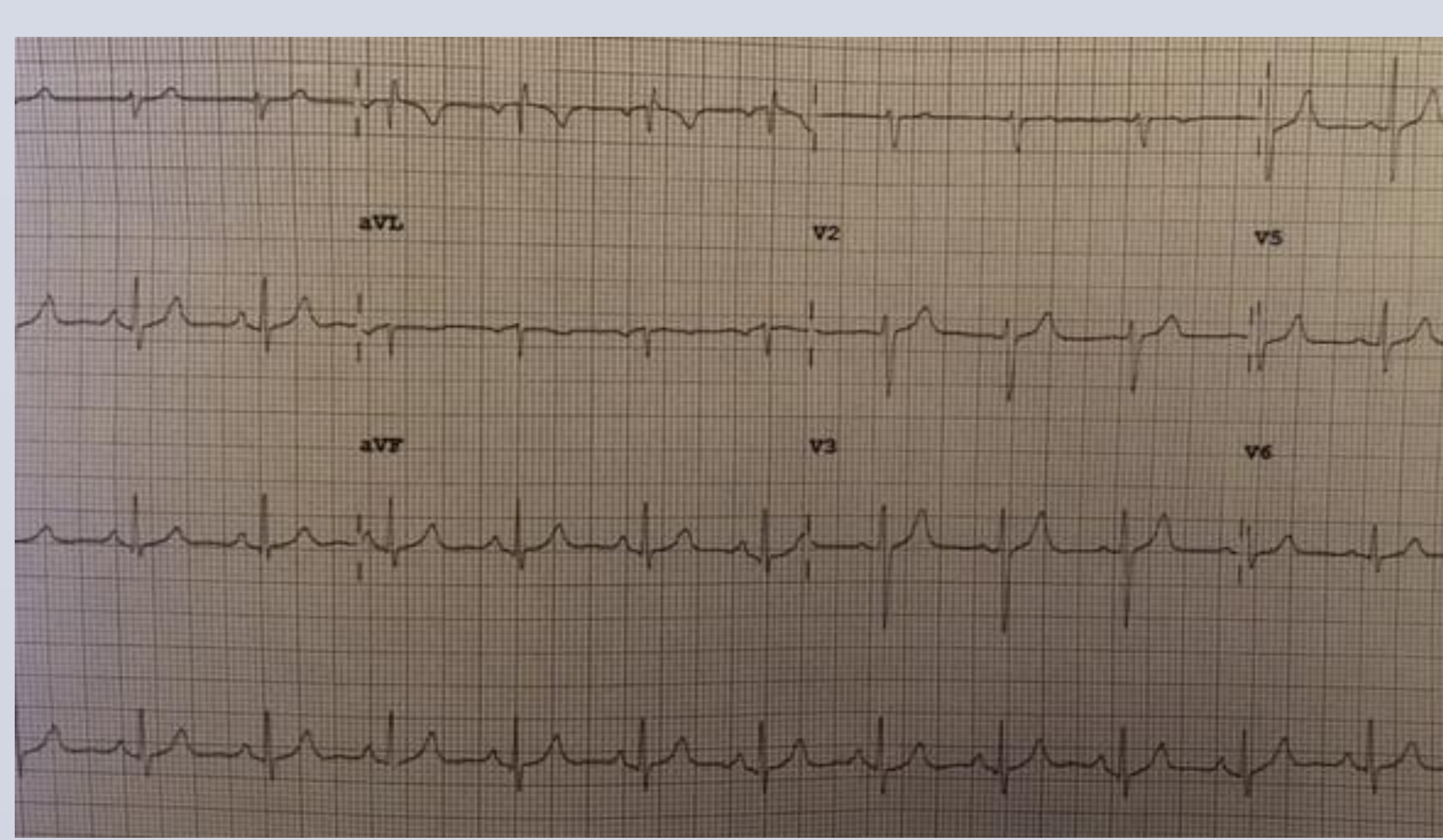

2. ECG on admission - QTC $530 \mathrm{msec}$

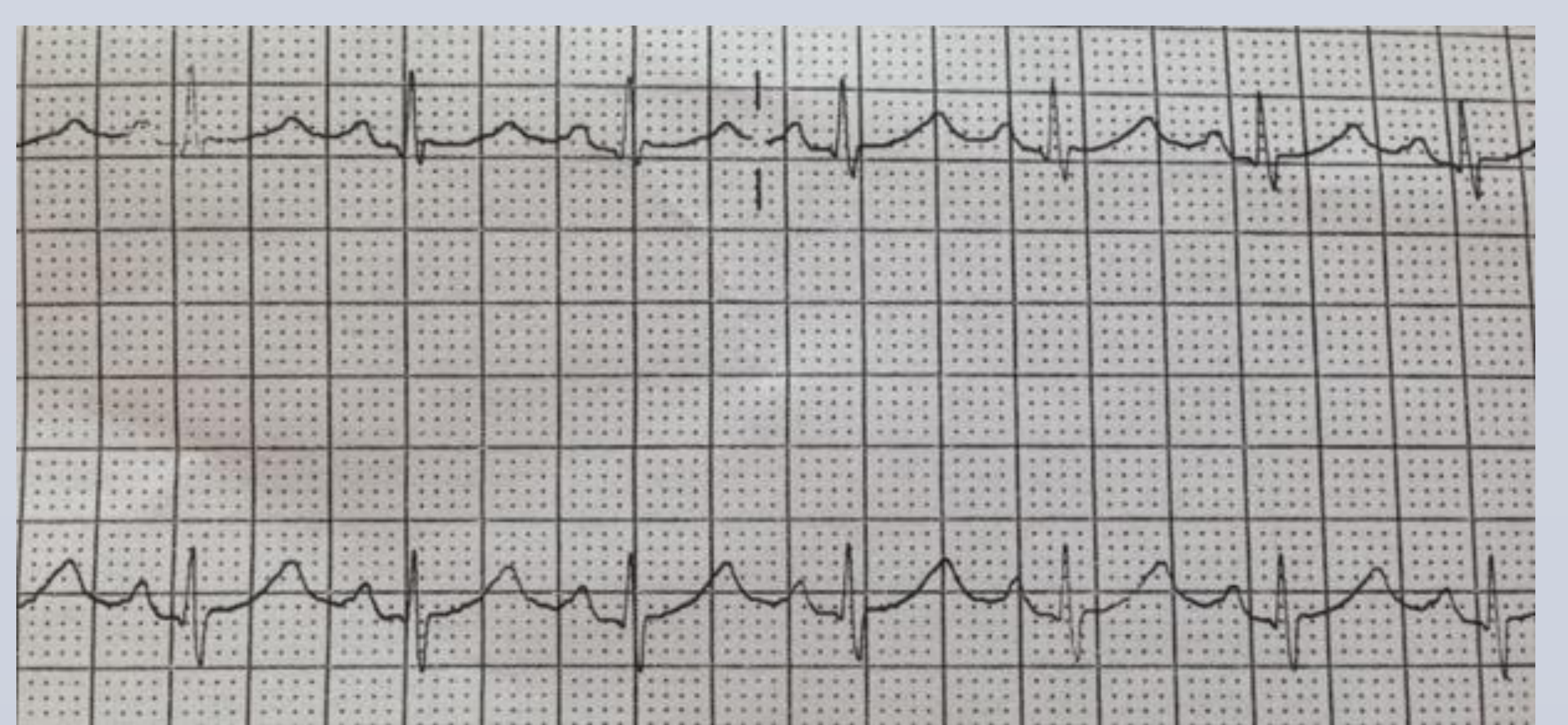

3. ECG at time of cardiac arrest

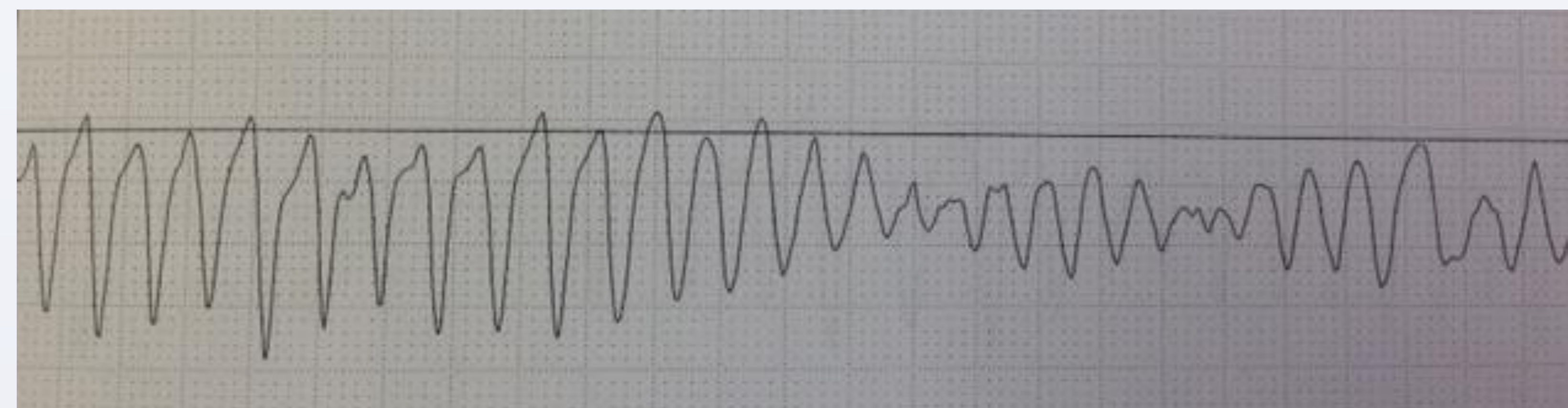

4. ECG 1 week post-arrest QTC 399msec

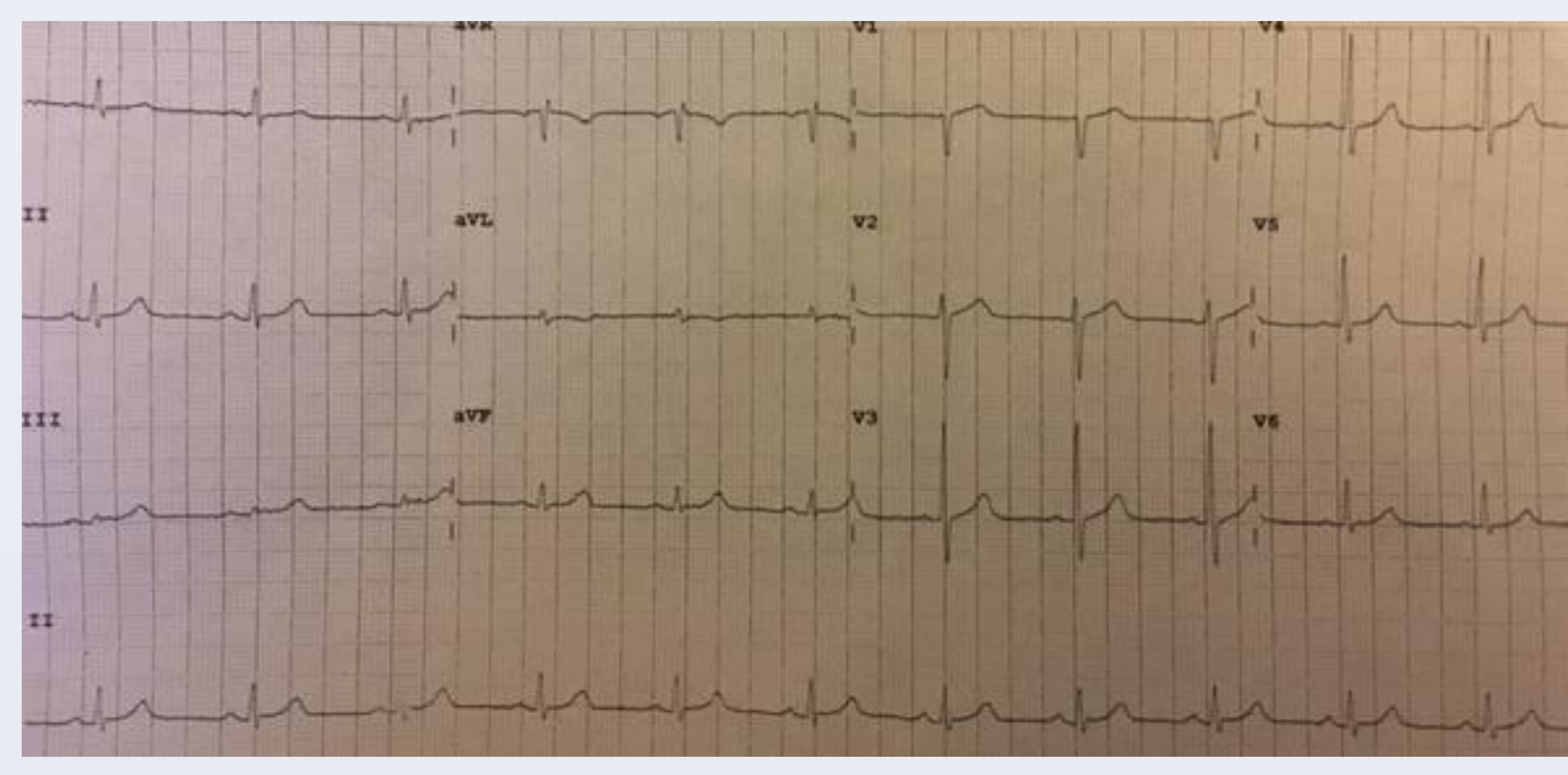

After a long stay on ITU followed by rehabilitation he made a good recovery and had a normal ECG and QTC at discharge.

\section{Discussion}

Glucocorticoid deficiency is known to decrease glucocorticoid inducible kinase-1 (SGK1) which controls expression of the hERG gene (human ether-a-go-go related gene). This in turn encodes the alpha subunit of the rapidly activating delayed rectifier potassium channel $\left(\mathrm{I}_{\mathrm{Kr}}\right)$ which mediates cardiac repolarisation. Hence there is delayed repolarisation with consequent long QT interval in glucocorticoid deficiency. ${ }^{3}$

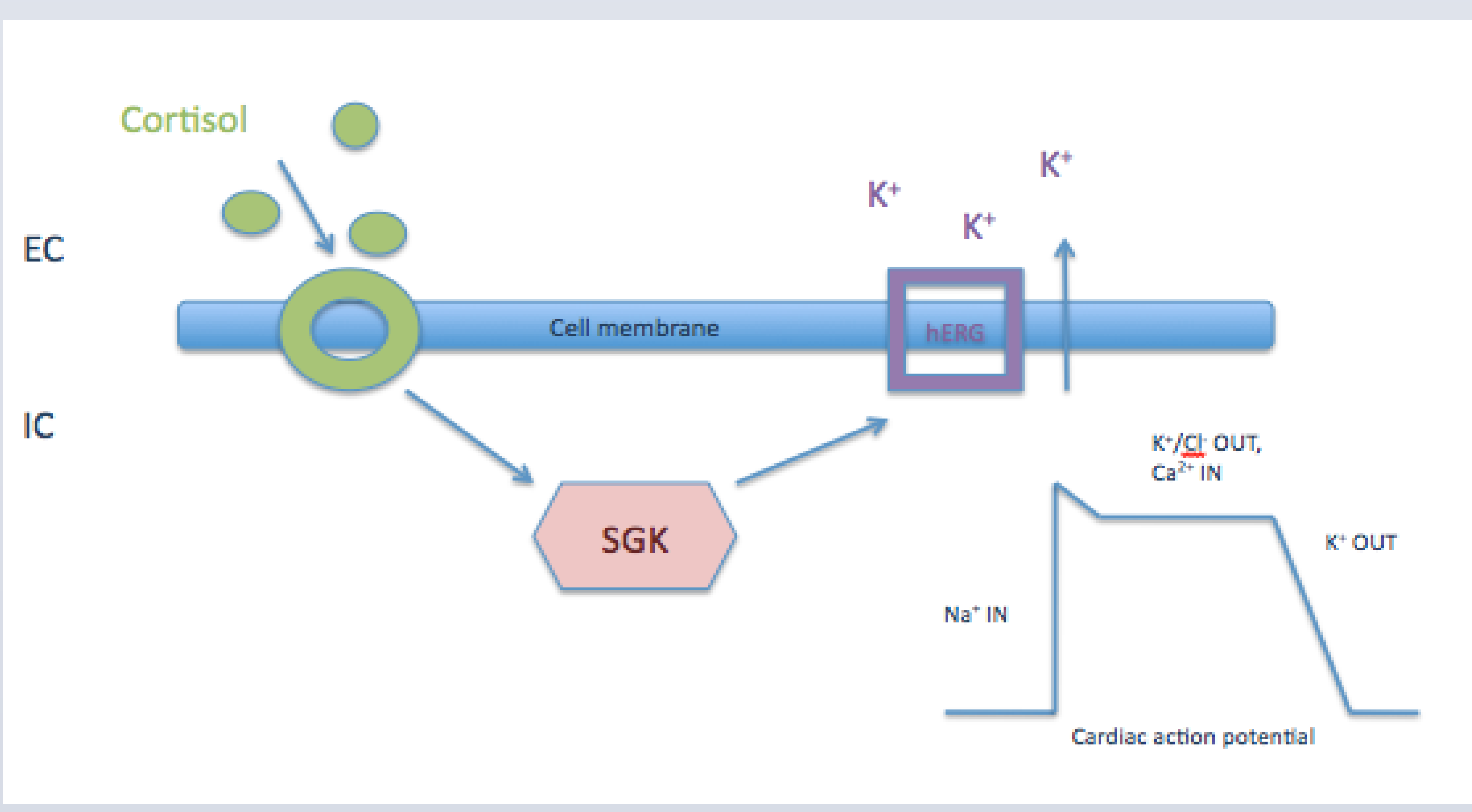

We believe this is the first reported case of life threatening arrhythmias associated with an acquired long QT interval in primary hypoadrenalism. The case is also unique with the arrhythmias occurring following correction of the long QT interval with treatment. Why these arrhythmias so rarely occur is unknown as is the reason for the preponderance of cases with secondary hypoadrenalism. Under reporting is a possibility as cardiac arrhythmias may have been attributed to electrolyte imbalance or coincidental cardiac disease rather than acquired long QT intervals.

\section{References}

1. Somerville, W. (1950) The effect of cortisone on the cardiogram in chronic adrenal insufficiency. British Medicak Journal 2: 860-862 2. Somerville, W., Levine, H.D. \& Thorn, G.W. (1951). The electrocardiogram in Addison's disease. Medicine (Baltimore) 30: 43-79. 3.Lamothe, S.M.\& Zhang, S. (2013) The Serum-Glucocorticoid-inducible Kinases SGK1 and SGK3 regulate hERG channel expression via Ubiquitin Ligase Nedd4-2 \&GTPase Rab11. The Journal of Biological Chemistry. 288:15075-15084.

\section{Contact}

Dr A Wilton Consultant Endocrinologist Ysbyty Gwynedd, Tony.Wilton@Wales.nhs.uk DrJ Rochester FY2 Ysbyty Gwynedd, Johanna.Rochester@Wales.nhs.uk 